\title{
The effect of second-generation antipsychotics on hippocampal volume in first episode of psychosis: Iongitudinal study
}

\author{
Michael Bodnar, Ashok K. Malla, Carolina Makowski, M. Mallar Chakravarty, Ridha Joober and \\ Martin Lepage
}

\section{Background}

Current neuroscience literature has related treatment with aripiprazole to improved memory performance and subcellular changes in the hippocampus.

\section{Aims \\ To explore the volumetric changes in hippocampal grey matter in people with a first episode of psychosis (FEP) treated with second-generation antipsychotics. \\ Method \\ Baseline and 1-year follow-up magnetic resonance images were obtained. Hippocampal volumes were estimated by using Freesurfer and MAGeT-Brain. Subgroups included: aripiprazole $(n=13)$, olanzapine $(n=12)$, risperidone/paliperidone $(n=24)$, refused-antipsychotics $(n=13)$ and controls $(n=44)$. \\ Results \\ Aripiprazole subgroup displayed significant increases in bilateral hippocampal volume compared with all other subgroups (FreeSurfer: all P's<0.012; MAGeT-Brain: all $P^{\prime} \mathrm{S}<0.040$ ).}

\section{Conclusions}

Aripiprazole is a first-line, second-generation treatment option that may provide an added benefit of pro-hippocampal growth. The biological underpinnings of these changes should be the focus of future investigations and may be key towards achieving a better clinical outcome for more individuals.

\section{Declaration of interest}

M.L. received financial assistance/compensation for research and educational events from Janssen-Ortho, Eli Lilly, Roche and Otsuka/Lundbeck Alliance. A.K.M. received financial assistance/compensation for research and educational activities from Pfizer, Janssen-Ortho, AstraZeneca and Bristol-Myers Squibb. R.J. received consultancy honorariums from Pfizer and Janssen-Ortho.

\section{Copyright and usage}

(c) The Royal College of Psychiatrists 2016. This is an open access article distributed under the terms of the Creative Commons Non-Commercial, No Derivatives (CC BY-NC-ND) licence.
In schizophrenia and the related psychoses, the most consistent longitudinal neuroimaging findings have highlighted progressive brain changes including marked decreases in whole-brain volume, whole-brain grey matter and frontal grey and white matter. ${ }^{1-3}$ However, a recent meta-analysis found whole-brain grey matter loss was less evident and, in some cases, actually increased in people with psychosis taking second-generation antipsychotics; the largest positive effect was found with clozapine. ${ }^{3}$ With reduced grey matter volume in the hippocampus linked to a poorer outcome, ${ }^{4,5}$ identifying a molecule that could be protective of an important brain structure such as the hippocampus could potentially alter the course of treatment and outcome. Although aripiprazole has been found to help improve memory performance in people with schizophrenia, ${ }^{6-8}$ it is uncertain whether there are any positive structural brain alterations, particularly in the hippocampus. As part of a longitudinal neuroimaging study investigating remission in a naturalistic-outcome setting, we explored hippocampal grey matter changes over a 1-year period. We compared people with a first episode of psychosis (FEP) taking aripiprazole with those taking other second-generation antipsychotics and with nonclinical controls. We hypothesised that those taking aripiprazole would show an increase in hippocampal volume.

\section{Method}

\section{Participants and treatment setting}

Individuals admitted and treated at the Prevention and Early Intervention Program for Psychoses (PEPP) aged 18-30 years with no history of neurological disease or head trauma causing loss of consciousness were eligible for the neuroimaging study.

PEPP is a specialised early intervention service offered at the Douglas Mental Health University Institute in Montreal, Canada. It serves 14- to 35-year-olds with a diagnosis of affective or nonaffective psychosis who have had no more than 1 month of previous antipsychotic treatment; without organic brain damage, a pervasive developmental disorder, an IQ below 70, or epilepsy; and do not have substance-induced psychosis. Diagnoses were determined using the Structured Clinical Interview for DSM-IV (SCID) ${ }^{9}$ and validated through consensus with a staff research psychiatrist. Treatment involved a comprehensive approach towards recovery with intensive medical and psychosocial management provided primarily through modified assertive case management. Pharmacotherapy for all patients, regardless of initial diagnosis, begins with a second-generation antipsychotic (olanzapine, risperidone, paliperidone, quetiapine or aripiprazole) within the recommended doses. If therapeutic response is not optimal within 4-6 weeks or significant side effects emerge, a different second-generation antipsychotic is prescribed. Although treatment for psychosis begins with an antipsychotic, patients who refuse drug therapy are still provided with all available psychosocial interventions, especially case management and family intervention. For complete programme details, see the work of Iyer et al. ${ }^{10}$

A control group for the neuroimaging study was recruited through advertisements in local newspapers; exclusion criteria included a current or past history of any Axis I disorder, any neurological disease, head trauma causing loss of consciousness or 
a first-degree relative diagnosed with schizophrenia or a related spectrum disorder.

After a comprehensive description of the study, written informed consent was obtained from all participants. All research was approved by the Research Ethics Board of the Douglas Mental Health University Institute and the McGill University Faculty of Medicine.

\section{Clinical and sociodemographic data}

Clinical data were collected near entry and at months 1, 2, 3, 6, 9, 12 and 18; baseline assessment occurred, on average, 9.4 days after entry (s.d.=8.8, range: 18-36). At each assessment, the type and dosage of antipsychotic prescribed were noted and converted into chlorpromazine equivalents. ${ }^{11,12}$ Data on education level (years completed), parental socioeconomic status, ${ }^{13}$ handedness, ${ }^{14}$ duration of untreated psychosis (DUP), duration of untreated illness (DUI) and full-scale $\mathrm{IQ}^{15,16}$ were obtained at baseline.

DUP was calculated as the period between the time of onset of psychotic symptoms (at the syndromal threshold based on the SCID) to adequate treatment with antipsychotics (defined as 30 days of continuous treatment or less if positive symptoms remitted). ${ }^{17}$ Any previous periods of psychosis which had resolved spontaneously were added to the total calculation of DUP, thus reflecting cumulative exposure to psychosis before receiving adequate treatment. Since its inception, PEPP has sought to reduce DUP (particularly delays in referral) by proactively promoting early case identification through outreach to the general community. DUI was defined as the time period from the onset of any psychiatric symptoms (anxiety, depression, suicidal ideation or social withdrawal) to adequate treatment with antipsychotics. ${ }^{18}$

Over the 18-month period, outcome was examined as changes in total positive symptoms from the Scale for the Assessment of Positive Symptoms (SAPS) ${ }^{19}$ and total negative symptoms from the Scale for the Assessment of Negative Symptoms (SANS). ${ }^{20}$ Also, the percentage of time spent in positive symptom remission during the interscan interval was calculated; positive remission was defined as mild or less on all four global scores of the SAPS. ${ }^{21}$ Evaluators, who are not involved in patient treatment, have established inter-class correlations of 0.89 and 0.71 on the SAPS and SANS respectively.

\section{Longitudinal structural magnetic resonance imaging (MRI) data acquisition and processing}

Scanning was completed at the Montreal Neurological Institute on a 1.5 T Siemens whole-body MRI system. For each participant, $T_{1}$ MR images were acquired using a $3 \mathrm{D}$ gradient-echopulse sequence (repetition time $=22 \mathrm{~ms}$; echo time $=9.2 \mathrm{~ms}$; flip angle $=30^{\circ}$; rectangular field of view $=256 \mathrm{~mm}$ superior-inferior $\times 204 \mathrm{~mm}$ anterior-posterior to the commissural plane; 180 sagittal slices; voxel size $\left.=1 \mathrm{~mm}^{3}\right)$. The same scanner and identical parameters were used at both Scan 1 (baseline) and Scan 2 (1-year follow-up); 88 people with FEP (treated between January 2004 and June 2014) and 46 controls completed both scans.

To obtain whole hippocampal volumes, $T_{1}$ images were automatically processed in FreeSurfer v5.3 (http://surfer.nmr.mgh.harvard. edu) using the hippocampal-subfields ${ }^{22}$ and longitudinal ${ }^{23,24}$ streams. The $T_{1}$ images were reprocessed using an independent pipeline, the MAGeT-Brain (Multiple Automatically Generated Templates) algorithm, ${ }^{25}$ based on subfield definitions derived from the Chakravarty Laboratory; ${ }^{26}$ see supplementary data material for a brief summary of this technique. The hippocampal labels derived from MAGeT-Brain were found to strongly overlap with labels manually derived as well as with those automatically derived using both FreeSurfer and FSL. ${ }^{27}$ Subfield volumes derived from each technique were summed to obtain left- and right-side hippocampal volumes; values from Scan 1 were subtracted from Scan 2 to present volumes as a function of change over time. Finally, total intracranial volume (TIV) was estimated using FreeSurfer for patients and controls.

\section{Defining antipsychotic treatment subgroups}

Patients were separated into subgroups based on the type of antipsychotic taken during the interscan interval. To be considered for a subgroup, a patient had to take one type of an antipsychotic for a minimum of 6 consecutive months with an average adherence above 50\% (subgroups' averages were 11.9 months and $88 \%$ adherence). The chosen time period of 6 months was justified by a seminal paper written by Lieberman and colleagues, which highlighted that the median discontinuation for antipsychotics was around the 6-month period, ${ }^{28}$ confirmed by an independent trial. ${ }^{29}$ Medication adherence $(0=$ never $(0 \%), 1=$ very infrequently (1$25 \%), 2=$ sometimes (26-50\%), 3=quite often (51-75\%), 4=fully (76-100\%)) was determined using a validated protocol based on composite information obtained from the patient, family members and treating team and has been shown to be as efficacious as pillcounting. ${ }^{30}$ Patients who outright refused antipsychotic treatment or had less than $50 \%$ adherence (score of 2 or less) were categorised into the 'refused-antipsychotics (APs)' subgroup.

\section{Statistical analyses}

Hippocampal volumes from FreeSurfer and MAGeT-Brain were analysed using a repeated-measures ANOVA with 'group' (risperidone/paliperidone, olanzapine, aripiprazole, refused-APs, controls) as the between-group factor and 'side' (left, right) as the within-group factor. Analyses were one-tailed and included the following covariates: age at Scan 1, age at Scan 2, education, gender, handedness, overall antipsychotic dosage per month and TIV.

Sample characteristics were analysed by using one-way ANOVAs for continuous variables or Kruskall-Wallis $H$-tests for nominal variables; DUP and DUI were analysed by using median tests. SAPS and SANS totals (at baseline, month 6, month 12, month 18) were analysed by using generalised estimating equations (GEEs); significant $P$-value was set to 0.012 (0.05/4 time points). GEE is a multivariate extension of the generalised linear model to analyse repeated measurements or other correlated observations. There are several advantages inherent to GEE for examining a large, longitudinal data-set including its robust nature to accommodate violations of normality (homogeneity of variance) and incomplete data based on population quantities and data distributions (allows the exclusion of a single missing observation without having to exclude an entire subject). All analyses were performed by using SPSS 22 (IBM Corporation, Armonk, New York, USA) and were two-tailed with a critical $P$-value of 0.05 , except where noted.

\section{Results}

\section{Sample size and subgroups}

Of the 88 people with FEP with two MRI scans, 26 were removed due to: missing key clinical data $(n=1)$, technical/processing errors $(n=1)$, concurrent antidepressant use $(n=5)$, polypharmacy of antipsychotics $(n=9)$ and insufficiently sized subgroups $(n=10$; see below for details). Two controls were also removed due to processing errors. The final sample size was 62 people with FEP and 44 controls.

Final FEP subgroups included: risperidone/paliperidone $(n=24)$; olanzapine $(n=12)$; aripiprazole $(n=13)$; and refused-APs $(n=13)$. Those taking quetiapine $(n=5)$, ziprasidone $(n=3)$, haloperidol $(n=1)$ and asenapine $(n=1)$ were excluded as any results would 
have been uninterpretable due to small subgroup sizes. Patients taking paliperidone $(n=5)$ were included as part of the risperidone/ paliperidone subgroup since paliperidone is the active metabolite of risperidone and has been shown to have a similar efficacy and treatment profile. ${ }^{31}$

\section{Sample characteristics}

For all participants, there were no significant between-group differences in age at Scan 1, age at Scan 2, parental socioeconomic status, gender, handedness, full-scale IQ, interscan interval or TIV; however, there were significant differences regarding education, with controls completing the most years. Among the FEP subgroups, there were no significant differences in the age at onset of psychosis, diagnosis, time from PEPP entry to Scan 1, DUP, DUI or antipsychotic dosage prescribed; however, there were significant differences regarding time spent on antipsychotic and overall medication adherence. As expected, the refused-APs subgroup spent the lowest time taking the prescribed antipsychotic (average of $3.4 v .11 .9$ months for the other three subgroups) and had the lowest adherence (average of $35 \%$ v. $88 \%$ for the other three subgroups). See Tables 1 and 2 for data and results.

\section{Symptom totals, symptom total changes and remission}

For SAPS total, there was a significant main effect of 'time' (Wald $\chi^{2}=188.24, \mathrm{df}=3, P<0.001$ ) (Fig. 1). All patients with FEP displayed a significant decrease from baseline to month $6(P<0.001)$ with no changes thereafter (all $P$ 's $>0.273$ ). Over the interscan interval, there were no significant subgroup differences in the change of positive symptom totals (Table 2). In addition, there were no significant subgroup differences in the amount of time spent in positive symptom remission (Table 2).

For SANS total, there was a significant main effect of 'time' (Wald $\chi^{2}=27.39, \mathrm{df}=3, P<0.001$ ) and of 'group' (Wald $\chi^{2}=11.14$, $\mathrm{df}=3, P=0.011$ ) (Fig. 1). All patients with FEP displayed a significant decrease from baseline to month $6(P<0.001)$ with minimal changes thereafter (all $P$ 's $>0.102$ ). The risperidone/paliperidone subgroup had a higher total overall compared with the other three subgroups (all $P$ 's $<0.041$ ); no other subgroup differences were apparent (all $P$ 's $>0.354$ ). Over the interscan interval, there were no significant subgroup differences in the change of negative symptom totals (Table 2).

\section{Hippocampal volume change}

For FreeSurfer, the repeated-measures ANCOVA revealed a significant between-group effect $\left(F_{4,94}=2.88, P=0.014\right)$. Further analyses revealed that the aripiprazole subgroup had a significantly larger increase in total bilateral volume compared with the risperidone/ paliperidone subgroup ( $P=0.008$, Cohen's $d=0.92$ ), the olanzapine subgroup $(P=0.005$, Cohen's $d=1.38)$, the refused-APs subgroup ( $P=0.005$, Cohen's $d=1.30)$ and the controls $(P=0.002$, Cohen's $d=1.20$ ).

For MAGeT-Brain, the repeated-measures ANCOVA revealed a significant between-group effect $\left(F_{4,94}=2.90, P=0.013\right)$. Further analyses revealed that the aripiprazole subgroup had a significantly larger increase in total bilateral volume compared with the risperidone/paliperidone subgroup ( $P=0.030$, Cohen's $d=0.60$ ), the olanzapine subgroup ( $P=0.003$, Cohen's $d=0.94)$, the refused-APs subgroup $(P=0.043$, Cohen's $d=0.51)$ and the controls $(P=0.018$, Cohen's $d=0.63)$. See Fig. 2 for results and supplementary Table DS1 for raw volumes.

\section{Correlations involving changes over interscan interval}

Partial correlations, controlling for subgroup, revealed no significant correlations between hippocampal volume change and SAPS and SANS total changes (Table 3). Exploring only within the aripiprazole subgroup ( $n=13$ ), bivariate correlations were also not significant: between SAPS total change and hippocampal volume change for FreeSurfer volumes $(r=0.376, P=0.206)$ or for MAGeT-Brain volumes $(r=0.291, P=0.334)$; between SANS total change and hippocampal volume change for FreeSurfer volumes $(r=-0.260, P=0.391)$ or for MAGeT-Brain volumes $(r=-0.203$, $P=0.506)$; or between total antipsychotic dosage and hippocampal volume change for FreeSurfer volumes $(r=-0.315, P=0.294)$ or for MAGeT-Brain volumes $(r=-0.236, P=0.268)$.

\section{Correlations at Scan 1 and Scan 2}

Partial correlations, controlling for subgroup, between SAPS total and hippocampal volumes at both Scan 1 and at Scan 2 revealed no significant associations for FreeSurfer volumes (all $P_{\mathrm{s}}>0.467$ ) or for MAGeT-Brain volumes (all $P$ 's $>0.643$ ). Partial correlations with SANS total at Scan 1 revealed significant negative associations with right $(r=-0.286, P=0.025)$ and total $(r=-0.267$, $P=0.037)$ FreeSurfer volumes; there were no significant correlations found for the MAGeT-Brain volumes (all $P$ 's $>0.175$ ). At Scan 2, there were no significant correlations between SANS total and hippocampal volumes for either FreeSurfer volumes (all $P$ 's $>0.420$ ) or for MAGeT-Brain volumes (all P's $>0.818$ ) (supplementary Table DS2).

\begin{tabular}{|c|c|c|c|c|c|c|c|c|}
\hline Variable & $\begin{array}{l}\text { Risperidone/ } \\
\text { paliperidone } \\
\quad(n=24)\end{array}$ & $\begin{array}{c}\text { Olanzapine } \\
(n=12)\end{array}$ & $\begin{array}{l}\text { Aripiprazole } \\
\qquad(n=13)\end{array}$ & $\begin{array}{l}\text { Refused-APS } \\
\qquad(n=13)\end{array}$ & Controls $(n=44)$ & Statistic & d.f. & $P$ \\
\hline Age at onset, years: mean (s.d.) & $22.5(3.5)$ & $22.3(3.2)$ & $23.3(4.1)$ & $24.3(3.1)$ & - & $F=0.94$ & 3.57 & 0.429 \\
\hline Parental SES, mean (s.d.) & $3.3(1.1)$ & $3.0(1.1)$ & $2.9(0.8)$ & $3.1(0.9)$ & $3.3(0.8)$ & $\chi^{2}=4.45$ & 4 & 0.348 \\
\hline Education, mean (s.d.) ${ }^{b}$ & $11.1(2.4)$ & $12.7(2.3)$ & $12.5(2.0)$ & $12.7(2.4)$ & $14.3(2.5)$ & $F=7.22$ & 4.101 & $<0.001$ \\
\hline Full-scale IQ, mean (s.d.) [n] & $96.5(14.6)$ & $100.8(16.5)$ & $105.5(13.9)$ & $105.5(12.5)$ & $111.1(14.6)[42]$ & $F=3.05$ & 4.95 & 0.233 \\
\hline Right handed, $n(\%)$ & $17(70.8)$ & $10(83.3)$ & $12(92.3)$ & $9(69.2)$ & $38(86.4)$ & $\chi^{2}=4.75$ & 4 & 0.314 \\
\hline Male, $n(\%)$ & $18(75.0)$ & $6(50.0)$ & $10(76.9)$ & $9(69.2)$ & $26(59.1)$ & $\chi^{2}=3.84$ & 4 & 0.429 \\
\hline Non-affective disorder, $n(\%)^{c}$ & $23(95.8)$ & $8(66.7)$ & $10(76.9)$ & $10(76.9)$ & - & $\chi^{2}=5.54$ & 3 & 0.136 \\
\hline DUP, weeks: median [n] & $18.6[23]$ & 17.8 & $12.8[10]$ & $20.6[11]$ & - & $\chi^{2}=2.08$ & 3 & 0.556 \\
\hline DUI, weeks: median [n] & 394.6 & 200.9 & 207.4 [9] & $255.3[11]$ & - & $\chi^{2}=5.87$ & 3 & 0.118 \\
\hline
\end{tabular}




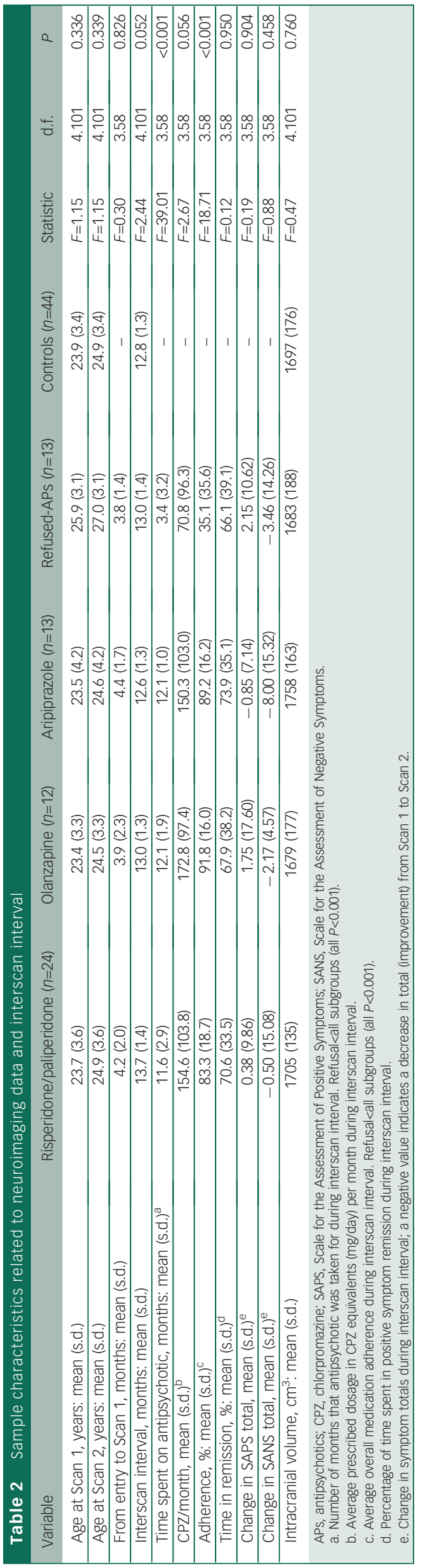

\section{Discussion}

In this naturalistic outcome study, we observed that people with FEP taking aripiprazole displayed a significant increase in bilateral hippocampal volume over a 1-year follow-up period compared with other people with FEP taking alternative second-generation antipsychotics and with non-clinical controls. This finding was observed with volumes obtained from two independent, fully automated neuroimaging processing pipelines (FreeSurferv5.3 and MAGeT-Brain).

Treatment with aripiprazole was equally efficacious compared with the other antipsychotics with respect to total positive and negative symptom reduction, and time spent in remission. These results suggest aripiprazole may be a good treatment option for people experiencing FEP with an added benefit of enhanced hippocampal plasticity. These results may have important clinical implications since hippocampal volume may vary as a function of future clinical status. ${ }^{4,5}$

\section{Aripiprazole and augmenting hippocampal growth}

The adult human brain is capable of producing new functional neurons from neural stem cells after postnatal development, but this growth is believed to be limited to the hippocampus. ${ }^{32}$ Adult neurogenesis has attracted even more attention, since hippocampal-dependent learning and memory, an enriched environment, and voluntary running have been shown to contribute to neurogenesis above and beyond normal daily growth. ${ }^{33}$ Therefore, the possibility exists for additional hippocampal growth if the correct pathways are triggered.

Aripiprazole has been described as a dopamine/serotonin system stabiliser due to its $\mathrm{D}_{2}, 5 \mathrm{HT}_{1 \mathrm{~A}}$ and $5 \mathrm{HT}_{7}$ agonistic and $\mathrm{D}_{1}, 5 \mathrm{HT}_{2 \mathrm{~A}}$ and $5 \mathrm{HT}_{6}$ antagonistic nature. ${ }^{34}$ Following administration of aripiprazole in animal models of depression and schizophrenia, better memory function, ${ }^{34-36}$ along with increased dopamine and brain-derived neurotrophic factor (BDNF) levels in the hippocampus, ${ }^{36,37}$ have been reported. Moreover, chronic exposure has led to increased proliferation of newly generated neurons in a mouse model involving neuronal loss in the dentate gyrus. ${ }^{38}$ In humans, improved memory function was identified in people with schizophrenia treated with aripiprazole. ${ }^{6-8}$ Moreover, a functional MRI study found improved working memory ability along with normalised activity in the anterior cingulate cortex (i.e. activation no longer differed from healthy controls) in people with schizophrenia who switched to aripiprazole. ${ }^{39}$ These findings suggest that aripiprazole may be related to an overall improvement of memory function as well as inducing neuroanatomical alterations in memory system structures.

Although the exact cellular mechanisms are not yet fully understood, accumulating evidence suggests that $5-\mathrm{HT}_{1 \mathrm{~A}}$ agonists may help induce adult neurogenesis. ${ }^{40}$ Antipsychotics with $5-\mathrm{HT}_{1 \mathrm{~A}}$ agonism include aripiprazole, ziprasidone, clozapine, asenapine and lurasidone. ${ }^{40}$ Treatments with clozapine or aripiprazole have been shown to enhance cell proliferation but not survival of newly generated neurons in the hippocampus. ${ }^{38,41,42}$ Moreover, clozapine has been shown to reverse the negative phencyclidine-induced effect on neurogenesis; ${ }^{43}$ aripiprazole may have a similar effect as it has been shown to ameliorate other phencyclidine-induced effects. ${ }^{35,44}$ Moreover, one study showed that people with reduced BDNF secretion, related to a 'val66met' polymorphism, displayed poorer memory performance along with abnormal hippocampal functioning. ${ }^{45}$ Intriguingly, aripiprazole has been shown to enhance BDNF levels in the rat hippocampus ${ }^{36}$ as well as in human neuroblastoma cells. ${ }^{46}$ Taken together, and with BDNF known to play an important role in the survival and development of neurons throughout life, ${ }^{47}$ sustained treatment with $5-\mathrm{HT}_{1 \mathrm{~A}}$ agonistic 

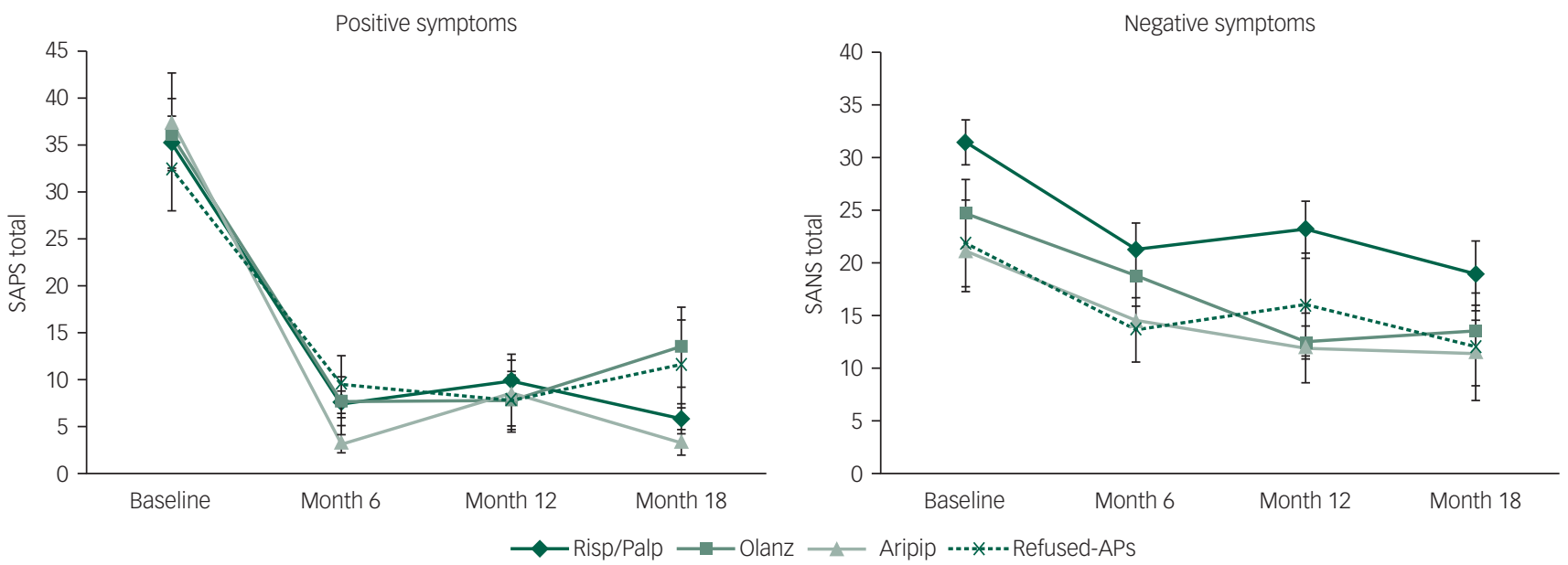

Fig. 1 Positive and negative symptom totals among first-episode psychosis (FEP) subgroups.

The error bars represent standard error. All people with FEP showed a significant improvement in both measures over the first 6 months of treatment. The risperidone/paliperidone (Risp/Palp) subgroup had higher negative symptoms overall compared with the other three subgroups. SAPS, Scale for the Assessment of Positive Symptoms; SANS, Scale for the Assessment of Negative Symptoms; Olanz, olanzapine; Aripip aripiprazole; APs, antipsychotics.

antipsychotics, such as aripiprazole, could favourably enhance adult neurogenesis in the hippocampus. ${ }^{40}$ Future studies are warranted to explore the underlying mechanisms of adult neurogenesis and the possible relationship with aripiprazole.

\section{Symptomatic changes in relation to hippocampal volume change}

In the current analysis, we observed that changes in hippocampal volume over the interscan interval did not significantly correlate with changes in either positive or negative symptoms or with the total amount of antipsychotics taken (in chlorpromazine equivalents). This potentially suggests that the change observed in hippocampal volume, namely in those taking aripiprazole, was not an effect related to symptomatic change over time or to the amount of medication taken. Of course, this claim should be verified in a future controlled study.

Moreover, we found no relationship between positive symptoms and hippocampal volumes at Scan 1 or at Scan 2 using both FreeSurfer and MAGeT-Brain. However, we did find a negative correlation between right hippocampal volume and negative symptoms total at Scan 1, using FreeSurfer volumes only. These findings are noteworthy, as previous studies using FreeSurfer have noted significant correlations with both positive ${ }^{48,49}$ and negative $e^{50}$ symptoms in hippocampal subfield analyses. For the positive symptoms, one study ${ }^{48}$ found these associations particular to the CA1 and CA2/3 subfields, another study ${ }^{49}$ found the associations particular to presubiculum and sublicuum subfields and the final study found no such relationship with hippocampal volumes. ${ }^{50}$

Of note, the study by Mathew et al ${ }^{49}$ explored hippocampal volumes in 886 participants across a six-site collaboration and noted an intersite scanning variation that was controlled for using a covariate. Although the power was evident in this study with such a large sample size, results may have been confounded by the varying scanners that were employed. An interesting suggestion would be to re-analyse their data using another pipeline such as MAGeT-Brain to help identify whether the issue was with FreeSurfer itself. $^{51}$

Finally, our results suggested that smaller hippocampal volumes at Scan 1 were related to worse negative symptoms, a finding supported by Kawano et al. ${ }^{50}$ In fact, this group showed the relationship was specific with $\mathrm{CA} 2 / 3$ and CA4/dentate gyrus subfield volumes. Although we did not explore subfield volumes, it would appear there are specific subfields that may be related to symptomology. As such, we further suggest that any future study exploring the relationship between aripiprazole and hippocampal growth should explore for changes within the subfields.

Our study, although not directly addressing cognitive benefits, does show that prolonged treatment with aripiprazole increased hippocampus volume in people with FEP who had limited or no previous exposure to antipsychotic medications. With the added benefit of stimulating adult neurogenesis above and beyond normal daily growth, aripiprazole could represent not only a pharmacological treatment for symptomatic management in psychosis, but could potentially help repair, in part, a putatively dysfunctional brain circuit in schizophrenia and the related psychoses.

\section{Limitations}

Our results are strengthened by the fact that our patients are largely previously untreated with antipsychotic medication, who are from a defined catchment area and treated in an early intervention service, not exclusively as in-patients, and, therefore, are truly representative of people with FEP with varying severity. Furthermore, we removed those who were taking multiple antipsychotics, antidepressants or mood stabilisers. However, there are a number of limitations to consider. To start, our study had two time points separated by 1 year, making it problematic to characterise the temporal characteristics of the hippocampal volume change. Second, the sample size of each FEP subgroup was relatively small which may have limited the generalisability of our findings. However, to date, our analysis represents the largest sized multigroup investigation of hippocampal change in relation to taking specific antipsychotic medications. ${ }^{3}$ Third, we did not systematically examine memory performance. Hence, it would be fundamental to confirm that memory is indeed enhanced following treatment with aripiprazole, and this improvement in performance may be related to a volumetric increase in the hippocampus. Fourth, although our patients demonstrated an overall medication adherence above $80 \%$ using a reliable and validated method, ${ }^{30}$ it was not possible to monitor the direct intake of medication or how adherence may have affected the results. Future studies may consider employing long-acting injectables to 

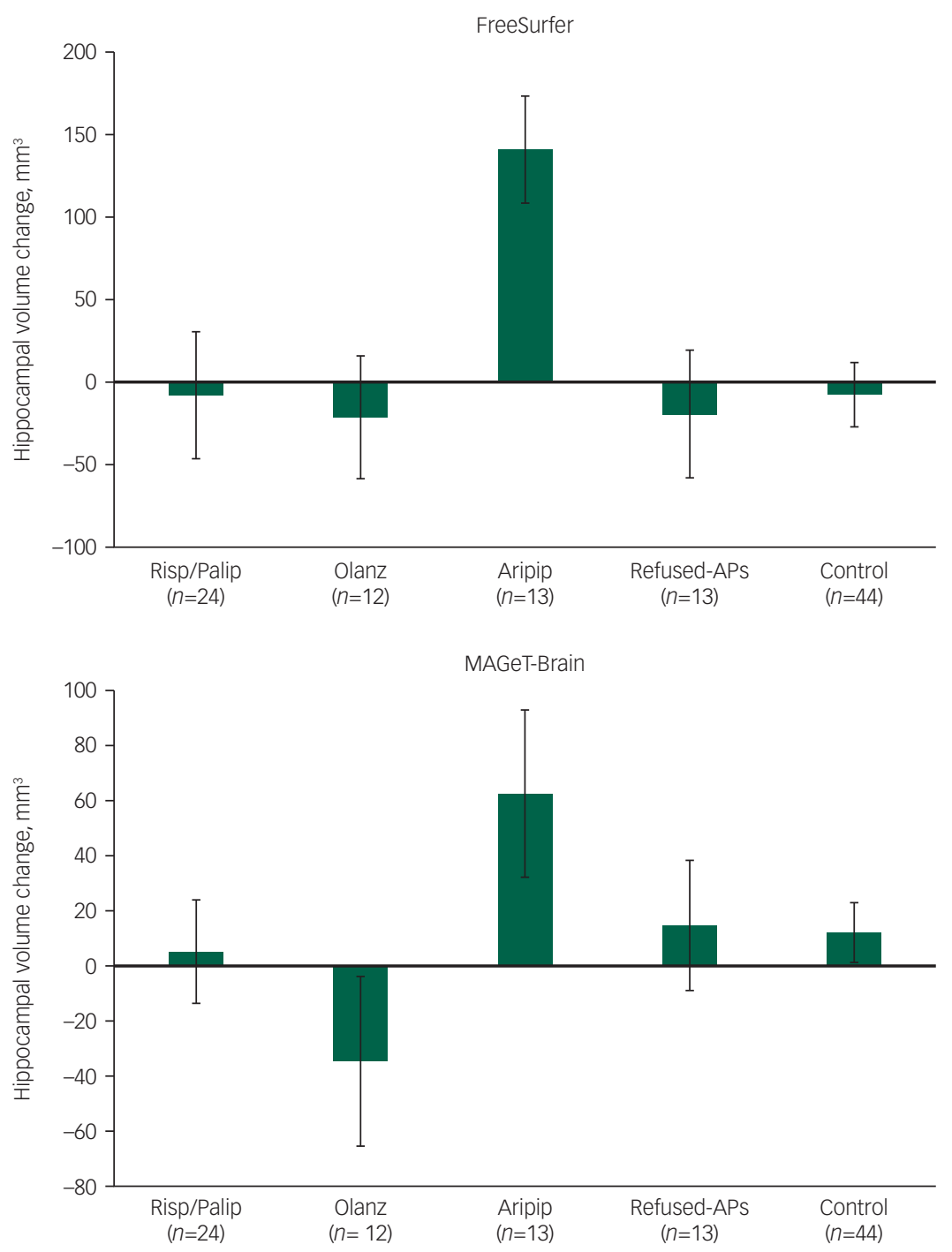

Fig. 2 Change in grey matter volume in the hippocampus for first-episode psychosis (FEP) subgroups and controls.

The error bars represent standard error. The aripiprazole (Aripip) subgroup displayed a significantly larger change in bilateral hippocampal volume compared with all FEP subgroups and controls for volumes derived from both Freesurfer and MAGeT-Brain. Risp/Palip, risperidone/paliperidone: Olanz, olanzapine; APs, antipsychotics

ensure adherence or measure medication plasma levels as an objective method to confirm actual medication adherence. Fifth, our study utilised 1.5 T MR images which limited the resolution to properly examine specific subfield volumetric changes. Moreover, there have been several issues raised with the FreeSurfer subfield estimations. ${ }^{51}$ Thus, this analysis focused on whole hippocampal volumes.
Finally, although a double-blind, randomised controlled trial would have maximised clarity of our results, this would not be practical in a naturalistic outcome research clinic. However, our current analysis does mimic a non-randomised, concurrent control (NRCC) trial. For clarity, an NRCC includes a control group (nonaripiprazole treated) that receives treatment at the same time as the intervention group (aripiprazole treated), but group assignation is

\begin{tabular}{|c|c|c|c|c|c|c|}
\hline & \multicolumn{2}{|c|}{ SAPS total change } & \multicolumn{2}{|c|}{ SANS total change } & \multicolumn{2}{|c|}{ CPZ total } \\
\hline & $r$ & $P$ & $r$ & P & r & P \\
\hline \multicolumn{7}{|l|}{ Freesurfer } \\
\hline Left change & -0.005 & 0.972 & -0.061 & 0.640 & 0.012 & 0.904 \\
\hline Right change & 0.077 & 0.554 & -0.093 & 0.474 & 0.083 & 0.398 \\
\hline Total change & 0.043 & 0.744 & -0.093 & 0.474 & 0.061 & 0.539 \\
\hline \multicolumn{7}{|l|}{ MAGeT-Brain } \\
\hline Left change & 0.108 & 0.408 & -0.082 & 0.531 & -0.131 & 0.184 \\
\hline Right change & 0.024 & 0.856 & 0.027 & 0.837 & 0.045 & 0.647 \\
\hline Total change & 0.080 & 0.542 & -0.036 & 0.783 & -0.055 & 0.578 \\
\hline
\end{tabular}


not random. An NRCC does have several advantages over a randomised controlled trial, and one stands out more than any other - it allows a clinic, such as ours, to continue to provide the best possible treatment to all patients. This follows as our entire treatment process included continual input from the clinician, the case manager, the patient and the patient's family. The act of random allocation within our clinic could have been met at odds with the treating team, the patient and/or family members, ultimately reducing the effectiveness of the service provided, thus decreasing study feasibility. Additionally, random allocation would have been met with involved increased treatment costs, the burden of keeping a double-blind study, and improbability of obtaining subsamples of maximum proportion derived from a defined catchment area. So, we do not believe, based on our clinic set-up and public healthcare system, that random assignment would have not been possible. But with our study strongly resembling an NRCC, we believe our results are on par with those that would have been obtained from a randomised controlled trial.

Michael Bodnar, PhD, Prevention and Early Intervention Program for Psychoses (PEPP - Montreal), Douglas Mental Health University Institute Montreal Canada; Department of Psychology, McGill University, Montreal, Canada; Ashok K. Malla, MD, Prevention and Early Intervention Program for Psychoses (PEPP - Montreal), Douglas Menta Health University Institute, Montreal, Canada; Department of Psychiatry, McGill University, Montreal, Canada; Carolina Makowski, BSc, Integrated Program in Neuroscience, McGill University, Montreal, Canada; M. Mallar Chakravarty, PhD, Department of Psychiatry, McGill University, Montreal, Canada; Integrated Program in Neuroscience, McGill University, Montreal, Canada; Ridha Joober, MD, PhD, Prevention and Early Intervention Program for Psychoses (PEPP - Montreal), Douglas Mental Health University Institute, Montreal, Canada; Department of Psychiatry, McGi University, Montreal, Canada; Martin Lepage, PhD, Prevention and Early Intervention Program for Psychoses (PEPP - Montreal), Douglas Mental Health University Institute, Montreal, Canada; Department of Psychology, McGill University, Montreal, Canada; Department of Psychiatry, McGill University, Montreal, Canada; Integrated Program in Neuroscience, McGill University, Montreal, Canada

Correspondence: Martin Lepage, PhD, Douglas Mental Health University Institute, Frank B. Common Pavilion, F1143, 6875 LaSalle Blvd., Verdun, Quebec H4H 1R3, Canada. Email: martin.lepage@mcgill.ca

First received 23 Nov 2015, final revision 9 Feb 2016, accepted 10 Feb 2016

\section{Funding}

The study is supported by operating grants from the Canadian Institutes of Health Research (CIHR; \#68961), the Sackler Foundation and Bristol-Myers Squibb to M.L./A.K.M. Salary awards include: Fonds de la Rechercheen Santé du Québec (FRSQ) (M.L., M.M.C. and R.J.) and Canada Research Chairs Program (A.K.M.). The funding sources had no role in study design; the collection, analysis nor interpretation of data; writing of the paper; nor in the decision for publication.

\section{Acknowledgements}

We thank PEPP-Montreal research staff for their help with recruitment and for conducting the clinical assessments, and all the people who participated in the study.

\section{References}

1 Moncrieff J, Leo J. A systematic review of the effects of antipsychotic drugs on brain volume. Psychol Med 2010; 40: 1409-22.

2 Olabi B, Ellison-Wright I, McIntosh AM, Wood SJ, Bullmore E, Lawrie SM. Are there progressive brain changes in schizophrenia? A meta-analysis of structural magnetic resonance imaging studies. Biol Psychiatry 2011; 70: 88-96.

3 Vita A, De Peri L, Deste G, Barlati S, Sacchetti E. The effect of antipsychotic treatment on cortical gray matter changes in schizophrenia: does the class matter? A meta-analysis and meta-regression of longitudinal magnetic resonance imaging studies. Biol Psychiatry 2015; 78: 403-12.

4 Bodnar M, Malla AK, Czechowska Y, Benoit A, Fathalli F, Joober R, et al. Neural markers of remission in first-episode schizophrenia: a volumetric neuroimaging study of the hippocampus and amygdala. Schizophr Res 2010; 122: 72-80.

5 Lieberman J, Chakos M, Wu H, Alvir J, Hoffman E, Robinson D, et al. Longitudina study of brain morphology in first episode schizophrenia. Biol Psychiatry 2001; 49 487-99.
6 Bervoets C, Morrens M, Vansteelandt K, Kok F, de Patoul A, Halkin V, et al. Effect of aripiprazole on verbal memory and fluency in schizophrenic patients: results from the ESCAPE study. CNS Drugs 2012; 26: 975-82.

7 Kern RS, Green MF, Cornblatt BA, Owen JR, McQuade RD, Carson WH, et al. The neurocognitive effects of aripiprazole: an open-label comparison with olanzapine. Psychopharmacology 2006; 187: 312-20

8 Riedel M, Spellmann I, Schennach-Wolff R, Musil R, Dehning S, Cerovecki A, et al. Effect of aripiprazole on cognition in the treatment of patients with schizophrenia. Pharmacopsychiatry 2010; 43: 50-7.

9 First MB, Gibbon M, Spitzer RL, Williams JBW, Benjamin LS. Structured Clinical Interview for DSM-IV Axis I Disorders, Research Version, Patient Edition. American Psychiatric Press, 1997

10 Iyer S, Jordan G, MacDonald K, Joober R, Malla A. Early intervention for psychosis: a Canadian perspective. J Nerv Ment Dis. 2015; 203: 356-64.

11 Woods SW. Chlorpromazine equivalent doses for the newer atypical antipsychotics. J Clin Psychiatry 2003; 64: 663-7.

12 Woods SW. C hlorpromazine Equivalent Doses for Atypical Antipsychotics: An Update 2003-2010. Available at: http://www.scottwilliamwoods.com/files/ WoodsEquivUpdate.doc (accessed 29 Feb 2016).

13 Hollingshead A. Two-Factor Index of Social Position. Yale University Press, 1965

14 Oldfield RC. The assessment and analysis of handedness: the Edinburgh inventory. Neuropsychologia 1971; 9: 97-113.

15 Wechsler D. Wecshler Adult Intelligence Scale (3rd edn). The Psychological corporation, 1997.

16 Wechsler D. Wechsler Abbreviated Scale of Intelligence. The Psychological Corporation, 1999.

17 Malla AK, Norman RM, Manchanda R, Townsend L. Symptoms, cognition, treatment adherence and functional outcome in first-episode psychosis. Psychol Med 2002; 32: 1109-19.

18 Malla A, Norman R, Schmitz N, Manchanda R, Bechard-Evans L, Takhar J, et al. Predictors of rate and time to remission in first-episode psychosis: a two-year outcome study. Psychol Med 2006; 36: 649-58.

19 Andreasen NC. Scale for the Assessment of Positive Symptoms (SAPS). University of lowa, 1984

20 Andreasen NC. Modified Scale for the Assessment of Negative Symptoms (SANS). University of lowa, 1984

21 Andreasen NC, Carpenter Jr WT, Kane JM, Lasser RA, Marder SR, Weinberger DR. Remission in schizophrenia: proposed criteria and rationale for consensus. Am J Psychiatry 2005; 162: 441-9.

22 Van Leemput K, Bakkour A, Benner T, Wiggins G, Wald LL, Augustinack J, et al. Automated segmentation of hippocampal subfields from ultra-high resolution in vivo MRI. Hippocampus 2009; 19: 549-57.

23 Reuter $M$, Fischl B. Avoiding asymmetry-induced bias in longitudinal image processing. Neurolmage 2011; 57: 19-21.

24 Reuter M, Schmansky NJ, Rosas HD, Fischl B. Within-subject template estimation for unbiased longitudinal image analysis. Neurolmage 2012; 61: 1402-18.

25 Chakravarty MM, Steadman P, van Eede MC, Calcott RD, Gu V, Shaw P, et al. Performing label-fusion-based segmentation using multiple automatically generated templates. Hum Brain Mapp 2013; 34: 2635-54.

26 Winterburn JL, Pruessner JC, Chavez S, Schira MM, Lobaugh NJ, Voineskos AN, et al. A novel in vivo atlas of human hippocampal subfields using high-resolution 3 T magnetic resonance imaging. Neurolmage 2013; 74: 254-65.

27 Pipitone J, Park MT, Winterburn J, Lett TA, Lerch JP, Pruessner JC, et al. Multi-atlas segmentation of the whole hippocampus and subfields using multiple automatically generated templates. Neurolmage 2014; 101: 494-512.

28 Lieberman JA, Stroup TS, McEvoy JP, Swartz MS, Rosenheck RA, Perkins DO, et al. Effectiveness of antipsychotic drugs in patients with chronic schizophrenia. N Engl J Med 2005; 353: 1209-23.

29 Stip E, Anselmo K. Effectiveness of antipsychotics: is the CATIE trial a tsunami? Can Fam Physician 2007; 53: 97-8.

30 Cassidy CM, Rabinovitch M, Schmitz N, Joober R, Malla A. A comparison study of multiple measures of adherence to antipsychotic medication in first-episode psychosis. J Clin Psychopharmacol 2010; 30: 64-7.

31 Fu DJ, Bossie CA, Sliwa JK, Ma YW, Alphs L. Paliperidone palmitate versus oral risperidone and risperidone long-acting injection in patients with recently diagnosed schizophrenia: a tolerability and efficacy comparison. Int Clin Psychopharmacol 2014; 29: 45-55.

32 spalding KL, Bergmann O, Alkass K, Bernard S, Salehpour M, Huttner HB, et al. Dynamics of hippocampal neurogenesis in adult humans. Cell 2013; 153 1219-27. 
33 Deng W, Aimone JB, Gage FH. New neurons and new memories: how does adult hippocampal neurogenesis affect learning and memory? Nat Rev Neurosci 2010; 11: 339-50.

34 Russo E, Citraro R, Davoli A, Gallelli L, Di Paola ED, De Sarro G. Ameliorating effects of aripiprazole on cognitive functions and depressive-like behavior in a genetic rat model of absence epilepsy and mild-depression comorbidity. Neuropharmacology 2013; 64: 371-9.

35 Nagai T, Murai R, Matsui K, Kamei $H$, Noda $Y$, Furukawa $H$, et al. Aripiprazole ameliorates phencyclidine-induced impairment of recognition memory through dopamine D1 and serotonin 5-HT1A receptors. Psychopharmacology 2009; 202 315-28.

36 Nowakowska E, Kus Kr, Ratajczak P, Cichocki M, Wozniak A. The influence of aripiprazole, olanzapine and enriched environment on depressant-like behavior, spatial memory dysfunction and hippocampal level of BDNF in prenatally stressed rats. Pharmacol Rep 2014; 66: 404-11.

37 Li Z, Ichikawa J, Dai J, Meltzer HY. Aripiprazole, a novel antipsychotic drug preferentially increases dopamine release in the prefrontal cortex and hippocampus in rat brain. Eur J Pharmacol 2004; 493: 75-83.

38 Yoneyama M, Hasebe $\mathrm{S}$, Kawamoto $\mathrm{N}$, Shiba T, Yamaguchi $\mathrm{T}$, Kikuta $\mathrm{M}$, et al. Beneficial in vivo effect of aripiprazole on neuronal regeneration following neuronal loss in the dentate gyrus: evaluation using a mouse model of trimethyltin-induced neuronal loss/self-repair in the dentate gyrus. J Pharmacol Sci 2014; 124: 99-111.

39 Schlagenhauf $F$, Dinges $M$, Beck $A$, Wustenberg $T$, Friedel $E$, Dembler $T$, et al Switching schizophrenia patients from typical neuroleptics to aripiprazole: effects on working memory dependent functional activation. Schizophr Res 2010; 118 . 189-200.

40 Schreiber R, Newman-Tancredi A. Improving cognition in schizophrenia with antipsychotics that elicit neurogenesis through 5-HT(1A) receptor activation. Neurobiol Learn Memory 2014; 110: 72-80.

41 Halim ND, Weickert CS, McClintock BW, Weinberger DR, Lipska BK. Effects of chronic haloperidol and clozapine treatment on neurogenesis in the adult rat hippocampus. Neuropsychopharmacology 2004; 29: 1063-9.

42 Chen $\mathrm{BH}$, Yan $\mathrm{BC}$, Park JH, Ahn JH, Lee $\mathrm{DH}$, Kim IH, et al. Aripiprazole, an atypical antipsychotic drug, improves maturation and complexity of neuroblast dendrites in the mouse dentate gyrus via increasing superoxide dismutases. Neurochem Res 2013; 38: 1980-8.

43 Maeda $K$, Sugino $H$, Hirose $T$, Kitagawa $H$, Nagai $T$, Mizoguchi $H$, et al. Clozapine prevents a decrease in neurogenesis in mice repeatedly treated with phencyclidine. J Pharmacol Sci 2007; 103: 299-308.

44 Gao J, Qin R, Li M. Repeated administration of aripiprazole produces a sensitization effect in the suppression of avoidance responding and phencyclidineinduced hyperlocomotion and increases D2 receptor-mediated behavioral function. J Psychopharmacol 2015; 29: 390-400.

45 Egan MF, Kojima M, Callicott JH, Goldberg TE, Kolachana BS, Bertolino A, et al. The BDNF val66met polymorphism affects activity-dependent secretion of BDNF and human memory and hippocampal function. Cell 2003; 112: 257-69.

46 Park SW, Lee JG, Ha EK, Choi SM, Cho HY, Seo MK, et al. Differential effects of aripiprazole and haloperidol on BDNF-mediated signal changes in SH-SY5Y cells. Eur Neuropsychopharmacol 2009; 19: 356-62.

47 Binder DK, Scharfman HE. Brain-derived neurotrophic factor. Growth Factors 2004; 22: 123-31.

48 Kuhn S, Musso F, Mobascher A, Warbrick T, Winterer G, Gallinat J. Hippocampal subfields predict positive symptoms in schizophrenia: first evidence from brain morphometry. Transl Psychiatry 2012; 2: e127.

49 Mathew I, Gardin TM, Tandon N, Eack S, Francis AN, Seidman $\sqcup$, et al. Medial temporal lobe structures and hippocampal subfields in psychotic disorders: findings from the Bipolar-Schizophrenia Network on Intermediate Phenotypes (B-SNIP) study. JAMA PSychiatry 2014; 71: 769-77.

50 Kawano M, Sawada K, Shimodera S, Ogawa Y, Kariya S, Lang DJ, et al. Hippocampal subfield volumes in first episode and chronic schizophrenia. PLOS One 2015; 10: e0117785.

51 Wisse LE, Biessels GJ, Geerlings MI. A critical appraisal of the hippocampal subfield segmentation package in freesurfer. Front Aging Neurosci 2014; 6: 261. 